Sabine Kuhangel

Der labyrinthische Text 


\section{Literaturwissenschaft/Kulturwissenschaft}

Herausgegeben von

Klaus-Michael Bogdal (Gerhard Mercator Universität Duisburg), Erhard Schütz (Humboldt-Universität zu Berlin), Jochen Vogt (Universität Essen)

In den Bänden dieser Reihe werden - ohne dogmatische Fixierung neuere methodische Entwicklungen der Literaturwissenschaft, insbesondere ihre kulturwissenschaftliche Neuakzentuierung reflektiert. Zentraler Gegenstandsbereich ist die deutschsprachige Literatur des 18. bis 20. Jahrhunderts in sozialgeschichtlicher, diskursanalytischer und narratologischer sowie kulturtheoretischer Perspektive. Ausblicke auf das Wirkungspotenzial publizistischer Formen, auf die Genres der 'Paraliteratur' und den Problemkreis ,Literatur in der Medienkonkurrenz' erweitern das thematische und methodische Spektrum. 
Sabine Kuhangel

\title{
Der labyrinthische Text
}

\author{
Literarische Offenheit \\ und die Rolle des Lesers
}

Deutscher Universitäts-Verlag 
Bibliografische Information Der Deutschen Bibliothek

Die Deutsche Bibliothek verzeichnet diese Publikation in der Deutschen Nationalbibliografie; detaillierte bibliografische Daten sind im Internet über <http://dnb.ddb.de> abrufbar.

Leicht überarbeitete Version der Dissertation Universität Bremen, 2002

Die Dissertation wurde finanziell gefördert durch die Stiftung Bildung und Wissenschaft im Stifterverband für die deutsche Wissenschaft.

\section{Auflage April 2003}

Alle Rechte vorbehalten

(C) Deutscher Universitäts-Verlag $\mathrm{GmbH}$, Wiesbaden, 2003

Lektorat: Ute Wrasmann / Anita Wilke

Der Deutsche Universitäts-Veriag ist ein Unternehmen der Fachverlagsgruppe BertelsmannSpringer.

wuww.duv.de

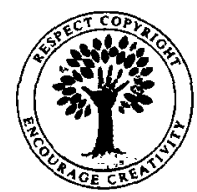

Das Werk einschließlich aller seiner Teile ist urheberrechtlich geschützt. Jede Verwertung außerhalb der engen Grenzen des Urheberrechtsgesetzes ist ohne Zustimmung des Verlags unzulässig und strafbar. Das gilt insbesondere für Vervielfältigungen, Übersetzungen, Mikroverfilmungen und die Einspeicherung und Verarbeitung in elektronischen Systemen.

Die Wiedergabe von Gebrauchsnamen, Handelsnamen, Warenbezeichnungen usw. in diesem Werk berechtigt auch ohne besondere Kennzeichnung nicht zu der Annahme, dass solche Namen im Sinne der Warenzeichen- und Markenschutz-Gesetzgebung als frei zu betrachten wären und daher von jedermann benutzt werden dürften.

Umschlaggestaltung: Regine Zimmer, Dipl.-Designerin, Frankfurt/Main

Gedruckt auf säurefreiem und chlorfrei gebleichtem Papier 


\section{Vorwort}

"One must be an inventor to read well."

R. W. Emerson

„Labyrinthische“ und für die Deutung ausgesprochen ,offene“ Texte laden die Leser in besonderer Weise zu interpretativer Kreativität ein. Die in diesen Texten vorherrschende Verwirrung und Bedeutungsvielfalt gilt es $\mathrm{zu}$ durchdringen und mittels individueller Deutung verstehbar zu machen. Mein Interesse an diesem Thema wurde erstmals während des Studiums geweckt, als ich mich mit Kafkas Roman Das Schloß auseinander setzte. Meine Faszination ist im Laufe der Jahre gewachsen und hat schließlich zur vorliegenden Studie geführt, die im Rahmen einer Promotion entstand. Ausgangspunkt meiner Untersuchung war eine Reihe von Werken, die wie Kafkas Schloß als labyrinthisch und außergewöhnlich offen (nachfolgend "selbstreflexiv offen") bezeichnet werden können. Im Laufe der Arbeit erwies es sich als unerlässlich, darüber hinaus der Offenheit aller literarischen Texte nachzugehen, d. h. literarische Offenheit im Allgemeinen mit Blick auf ihre Ursachen, Implikationen und Grenzen aus theoretischer Sicht auszuloten. Dies brachte notwendigerweise eine Analyse der Rolle des Lesers mit sich, denn Offenheit ist bei allen literarischen Werken - auch wenn sie oft nur minimal ausgeprägt ist auf eine Aktivierung der Leser ausgerichtet. Die Offenheit literarischer Werke kann als Einladung zu Interpretationsfreiheit und Interpretationsvielfalt verstanden werden.

Offenheit erweist sich nicht nur als eines der elementaren Themen der Literaturwissenschaft, sondern ihr kommt auch in anderen Zusammenhängen große Bedeutung zu. Sie steht in direkter Verbindung mit zentralen Fragen, die sich in den Bereichen Kultur, Politik und Wissenschaft in mitunter drängender Weise stellen. Das vorliegende Buch ist zwar auf die Literaturwissenschaft bezogen, jedoch soll es damit seinen speziellen Beitrag zur Erkundung eines umfassenderen Phänomens leisten, das meines Erachtens zu den bedeutendsten Themen unserer Zeit gehört. 
Ich möchte mich an dieser Stelle bei der Stiftung Bildung und Wissenschaft (im Stifterverband für die Deutsche Wissenschaft) für die finanzielle Unterstützung meiner Promotion bedanken.

Mein ganz besonderer Dank gilt meinem Doktorvater Herrn Professor Dr. Gert Sautermeister (Universität Bremen) für die Betreuung und Unterstützung dieses Projektes, für die Ermunterung zu diesem Thema sowie für seine eingehende und detaillierte Auseinandersetzung mit meiner Arbeit. Des Weiteren danke ich Herrn Professor Dr. Gerhard Pasternack (Universität Bremen) für seine kritischen und äußerst anregenden Kommentare.

Moreover, I would like to express my sincerest gratitude to Professor Jonathan Usher (University of Edinburgh) for his consistently useful advice and his practical assistance.

And heartfelt thanks to Steven for ... everything.

Sabine Kuhangel 


\section{Inhaltsverzeichnis}

Vorwort

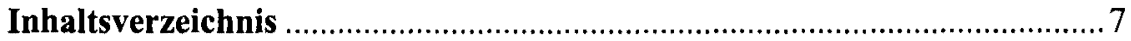

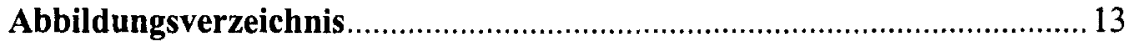

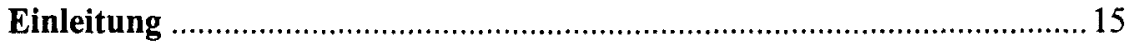

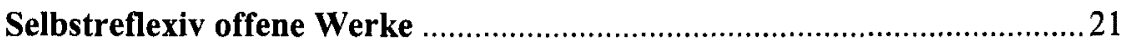

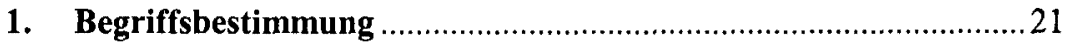

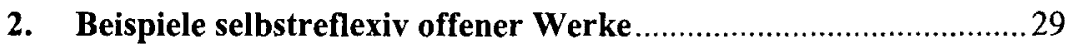

2.1 Selbstreflexiv offene Werke (physisch abgeschlossen) ................30

2.1.1 James Joyce: Ulysses ........................................................ 30

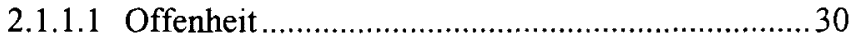

2.1.1.2 Die Rolle des Lesers ......................................... 33

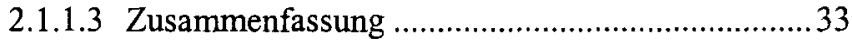

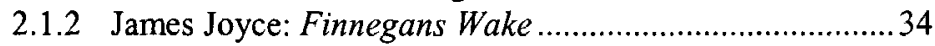

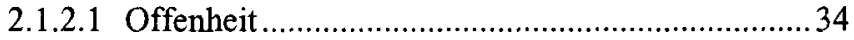

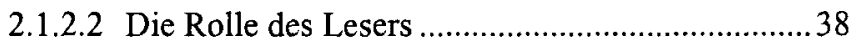

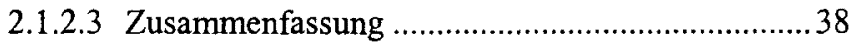

2.1.2.4 Kritische Anmerkungen zu Finnegans Wake........39

2.1.3 Franz Kafka: Der Proceß ............................................40

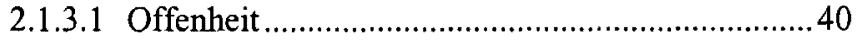

2.1.3.2 Die Rolle des Lesers ..............................................57

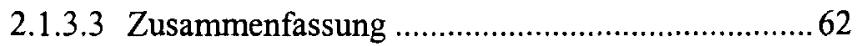

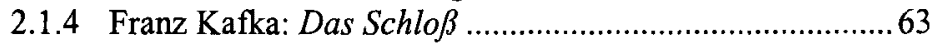

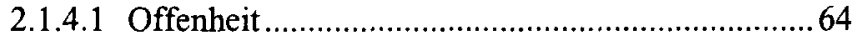

2.1.4.2 Die Rolle des Lesers .......................................... 79

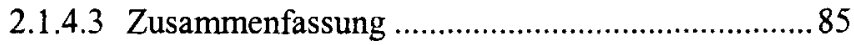

2.1.5 Samuel Beckett. Die Trilogie

(Molloy, Malone Dies, The Unnamable) ........................8 85

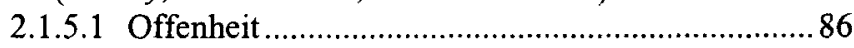

2.1.5.2 Die Rolle des Lesers .........................................94

2.1.5.3 Zusammenfassung ............................................ 95 
2.2 Selbstreflexiv offene Werke in Bewegung (physisch unabgeschlossen) .......................................................96

2.2.1 Julio Cortázar. Rayuela. Himmel-und-Hölle ....................96

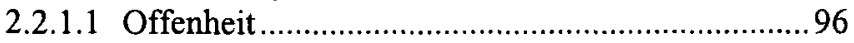

2.2.1.2 Die Rolle des Lesers: ....................................... 101

2.2.1.3 Metafiktionale Aspekte .....................................102

2.2.1.4 Zusammenfassung .............................................106

2.3 Selbstreflexiv offene Werke - Zusammenfassung ................... 107

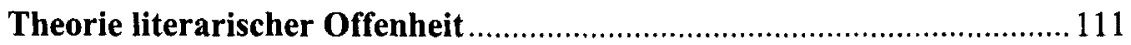

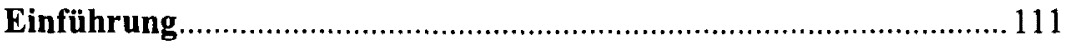

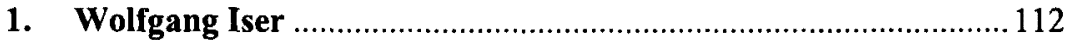

1.1 Beitrag zur theoretischen Bestimmung literarischer Offenheit. 113

1.1.1 Die Unbestimmtheit fiktionaler Texte und die Rolle des Lesers

1.1.1.1 Unbestimmtheit: Leerstellen zwischen

Textsegmenten

1.1.1.2 Die Rolle des Lesers: Freiheit bei der

Gestaltbildung

1.1.1.3 Der Unterschied zwischen fiktionalen und

nicht-fiktionalen Texten: unterschiedliche

Textstrategien

1.1.2 Die Grenzen der Unbestimmtheit und die Rolle des

Lesers

1.1.3 Die Dialektik von Unbestimmtheit und Bestimmtheit und die Rolle des Lesers.

1.1.4 Ausprägung von Unbestimmtheit als Unterscheidungsmerkmal.

1.2 Probleme der Theorie Isers

1.2.1 Unbestimmtheit als Form der Leserlenkung:

Ein Missverständnis?

1.2.1.1 Leserlenkende Funktion der Unbestimmtheit ..... 121

1.2.1.2 Leserlenkende Funktion der Leerstellen .............. 124

1.2.1.3 Isers Verständnis der eigenen Theorie ............... 126

1.2.2 Überstrapazierung des Begriffs der Leerstelle ................ 127

1.2.3 Leserlenkende Funktion des impliziten Lesers .............. 129

1.2.4 Unbestimmtheit der Textsegmente:

Ein missachteter Aspekt.

1.3 Zusammenfassung 
2. Umberto Eco 134

2.1 Das offene Universum der Kultur und die Grenzen der Interpretation: Grundlagen einer Theorie der Semiotik. 135

2.1.1 Kultur als Enzyklopädie: Voraussetzungen der Offenheit der Zeichen. 135

2.1.2 Die Offenheit der Zeichen: Unbegrenzte Semiose

2.1.3 Die Grenzen der Interpretation.

2.1.4 Das Wesen kommunikativer Prozesse

2.1.4.1 Enzyklopädie und die Auswahl von Codes......... 142

2.1.4.2 Die eingeschränkte Geltung des Begriffs des Codes: Ein kurzer Kommentar.

2.2 Die Offenheit ästhetischer Texte und die Grenzen der Interpretation

2.2.1 Vorbemerkungen

2.2.2 Die Offenheit ästhetischer Texte und die Rolle des Lesers

2.2.2.1 Offenheit ästhetischer Texte: Abweichung vom Code und unverbundenes Textmaterial

2.2.2.2 Die Rolle des Lesers: Einführen neuer Codes .... 148

2.2.2.3 Kritik: Abweichung vom Code nicht als Ursache von Offenheit

2.2.3 Die Offenheit der Zeichen in ästhetischen Texten 154

2.2.3.1 Offenheit der Zeichen: Voraussetzungen ästhetischer Offenheit...................................154

2.2.3.2 Zeichen mit besonderem Offenheitspotential ..... 155

2.2.4 Die Grenzen der Offenheit und die Rolle des Lesers..... 167

2.2.4.1 Grenzen der Offenheit: Kotext........................... 167

2.2.4.2 Die Rolle des Lesers: Grenzen beim Einführen neuer Codes

2.2.5 Die Dialektik zwischen Offenheit und Geschlossenheit und die Rolle des Lesers.

2.2.5.1 Die Dialektik des Textes und die Leserrolle ....... 169

2.2.5.2 Ecos Überbetonung der Grenzen der Interpretation: Eine Fehldeutung?

2.2.6 Der Modell-Leser.

2.2.6.1 Begriffsbestimmung............................................. 171

2.2.6.2 Problematik der Begriffsbestimmung ................. 171

2.3 Ausprägung von Offenheit als Unterscheidungsmerkmal ......... 174

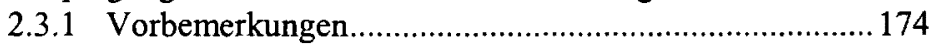

2.3.2 Offene und geschlossene Texte................................... 175 
2.3.3 Der Modell-Leser offener und geschlossener Texte ...... 180

2.3.3.1 Begriffsbestimmung.......................................... 180

2.3.3.2 Problematik der Begriffsbestimmung ................ 181

2.3.4 Die Metapher des Labyrinths ......................................... 184

2.3.5 Offene und geschlossene Texte - kein Werturteil.......... 187

2.3.6 Auflösung des Unterschieds zwischen offenen und geschlossenen Texten? .............................................. 189

2.4 Offenheit im Text und Öffnung von außen .............................191

2.4.1 Interpretation und Gebrauch ........................................ 191

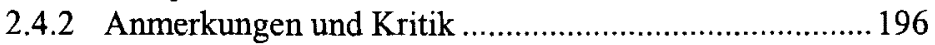

2.4.2.1 Semantische und kritische Interpretation:

Eine erhellende Unterscheidung.................. 196

2.4.2.2 Kritische Interpretation und (ideologie-)kritische

Interpretation: Problem der Begriffe ............. 197

2.4.2.3 Verwirrende Darstellung der Begriffe ................. 198

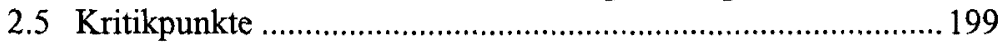

2.5.1 Leserlenkende Funktion des Modell-Lesers..................199

2.5.2 Das Problem der zwei Offenheiten im ästhetischen

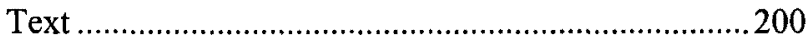

2.5.2.1 Emotive und semantische Offenheit in Ecos Frühwerk ......................................................200

2.5.2.2 Emotive und semantische Offenheit im Rahmen der Semiotik ..................................................202

2.5.3 Autoreflexivität und Offenheit .....................................204

2.5.4 Eigenarten und Probleme der Schriften Ecos.................206

2.5.4.1 Unklare Darstellungen ......................................206

2.5.4.2 Das Besondere im Dienst des Allgemeinen........206

2.5.4.3 Der labyrinthische Charakter der Theorie Ecos..207

2.6 Zusammenfassung.... 208

3. Zusammenführung der Theorien Isers und Ecos........................211

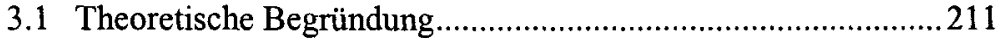

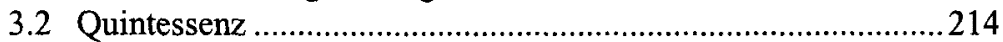

3.2.1 Offenheit der Zeichen ...............................................214

3.2.2 Offenheit durch Leerstellen............................................214

3.2.3 Zeichen mit besonderem Offenheitspotential..................215

3.2.4 Ausprägung von Offenheit als Unterscheidungs-

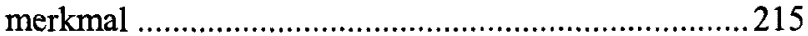

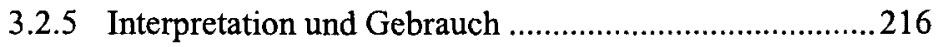


4. Anwendung der theoretischen Erkenntnisse auf die Beispieltexte

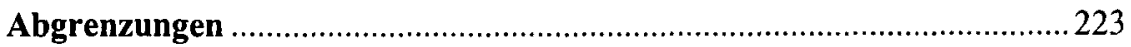

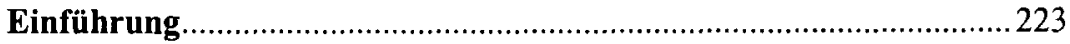

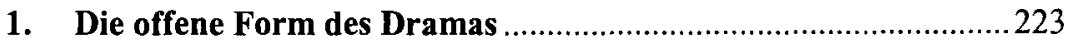

1.1 Die offene Form des Dramas..................................................224

1.1.1 Offenheit durch innere Vielheit?................................227

1.1.2 Offenheit durch Unbegrenztheit nach außen? ................229

1.2 Das epische Drama Brechts................................................... 230

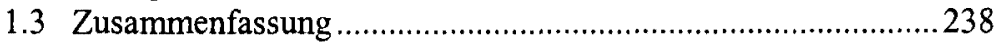

2. Selbstreflexive Offenheit:

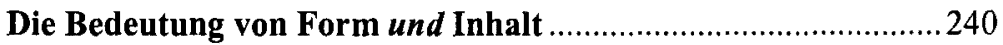

2.1 Surrealistische Texte ...............................................................241

2.2 Alain Robbe-Grillet: Die Jalousie oder die Eifersucht

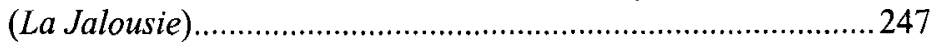

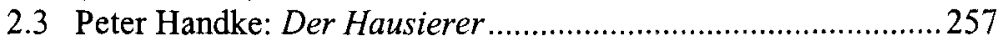

2.4 Zusammenfassung..............................................................2261

3. Selbstreflexive Offenheit in Werken in Bewegung:

Die Bedeutung von Form und Inhalt ..........................................263

3.1 B. S. Johnson: The Unfortunates ...........................................263

3.2 Andreas Okopenko: Lexikon-Roman ....................................266

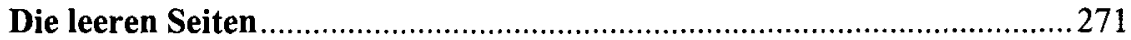

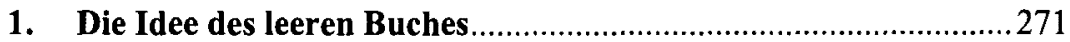

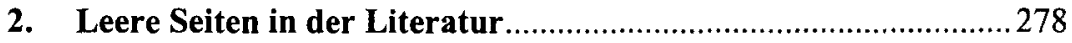

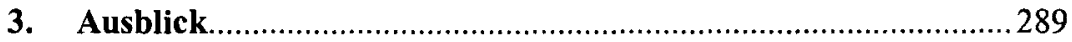

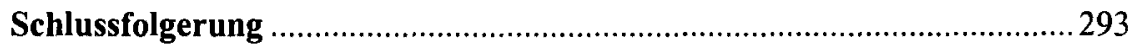

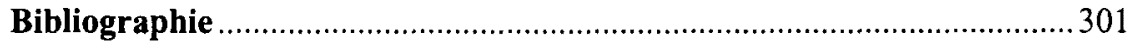




\section{Abbildungsverzeichnis}

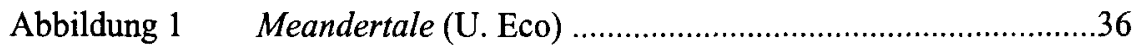

Abbildung 2 Relativity (M. C. Escher) ..................................................109

Abbildung $3 \quad$ Geschlossene und offene Texte (U. Eco) ........................... 178 